\title{
Factors in the Probability of COVID-19 Transmission in University Classrooms
}

Charles Connor

University of South Florida, cbconnor@usf.edu

Follow this and additional works at: https://digitalcommons.usf.edu/numeracy

Part of the Influenza Humans Commons, Interprofessional Education Commons, Occupational Health and Industrial Hygiene Commons, Scholarship of Teaching and Learning Commons, and the Virus Diseases Commons

\section{Recommended Citation}

Connor, Charles. "Factors in the Probability of COVID-19 Transmission in University Classrooms." Numeracy 13, Iss. 2 (2020): Article 7. DOI: https://doi.org/10.5038/1936-4660.13.2.1368 


\title{
Factors in the Probability of COVID-19 Transmission in University Classrooms
}

\begin{abstract}
University students and faculty members need an effective strategy to evaluate and reduce the probability that an individual will become infected with COVID-19 as a result of classroom interactions. Models are developed here that consider the probability an individual will become infected as a function of: prevalence of the disease in the university community, number of students in class, number of class meetings, and transmission rate in the classroom given the presence of an infected individual. Absolute probabilities that an individual will become infected in a classroom environment cannot be calculated because some of these factors have unknown values. Nevertheless, models suggest that some strategies for minimizing probability of infection are more effective than others. Given that COVID-19 might be present among faculty and students in the university community, limiting class meetings and class size are not likely effective strategies unless these numbers are dramatically reduced. That is, it is likely that infected individuals will be present in classrooms at some point during the term due to the large number of interactions among university faculty and students. The probability of infection of an individual in a classroom setting appears to be most sensitive to the effectiveness of transmission in the classroom, given the presence of an infected individual, especially if the likelihood of transmission itself is a function of class size. If on-campus instruction takes place, efforts should focus on reducing the probability of transmission through physical modifications and upgrades to classrooms and by social distancing measures.
\end{abstract}

\section{Keywords}

COVID-19, classroom, class size, university, model, quantitative literacy, numeracy, pandemic

\section{Creative Commons License} (c) (i) (8)

This work is licensed under a Creative Commons Attribution-Noncommercial 4.0 License

\section{Cover Page Footnote}

C. Connor is a professor of geology at the University of South Florida, former Chair of the Geology Department and former Associate Dean of Research for the College of Arts and Sciences. He studies volcanoes mostly, especially how to forecast volcanic eruptions and their hazards. Connor teaches students about quantitative methods in geology and about writing computer code to solve geology and geophysics problems. 


\section{Introduction}

Currently, in the summer of 2020, university faculty are wrestling with how to handle teaching during the coming Fall term and perhaps beyond, given the COVID-19 pandemic. Possible teaching strategies include online only; normal classroom instruction; hybrid models that offer reduced classroom interactions and some online instruction; and a variety of permutations of these options. Faculty members, including instructors and adjuncts, are asked to weigh the cost-benefit of generally more effective teaching in person against the unknown probability that they will contract the disease or worse yet spread it among their students by hosting classroom lectures and activities (Levantovskaya 2020). University students must evaluate if it is best for them to enroll or postpone enrollment, either because online courses are less effective or because the risks of attending regular class meetings appear to be too great. Administrators are faced with even broader issues, weighing encouragement of students to register against the liability of doing so (Kafka 2020).

Like all university faculty members, I am faced with making decisions about my teaching in the near future. Is online-only teaching necessary or overly conservative? Will the health and safety of students improve substantially from a hybrid teaching model, or not? How are updated epidemiological models about the rate of infection in the broader community best used to understand risks in specific classrooms? Like most faculty members, I am not a virologist or epidemiologist, but nevertheless I feel compelled to reach an informed and reasoned perspective for the sake of my students and the broader university community. This dilemma illustrates how critical quantitative literacy is to our professional and personal lives (Vacher and Wallace 2008; Wallace 2019; Vacher and Grawe 2019). I would like to rely on experts, but frankly epidemiologists currently have an overwhelming number of issues to resolve. Meanwhile, the time-frame is short for decisions about teaching in the Fall. In these circumstances, non-experts, such as myself, turn to their own quantitative literacy to consider how to react to new information from experts and to make sensible choices.

As with all natural hazards, there is great uncertainty in making decisions about teaching in a pandemic, but our understanding of the trade-offs can be improved by assessing the hazard quantitatively with a probability model (e.g., Connor 2011). In the following, I develop simple models for the probability of an individual becoming infected as a result of classroom activities during the term (Fall semester of 2020). Simple models are warranted because some factors that affect this probability are unknown. For example, there is currently great uncertainty about the rate of infection among communities (Manski and Molinari 2020). Even in the absence of robust probability estimates, models are useful because they provide an important way to evaluate the relative importance of different factors. These differences can inform our decisions about how to teach during the pandemic. 


\section{Simple Model}

Assume that the probability $(P)$ that one or more individuals (students or faculty members) will be infected by another individual in a classroom or laboratory setting during the term depends on these factors:

1. $\lambda$ - The prevalence of infection in the university population on a given day, assumed to be constant for the term. This prevalence of infection is best estimated from expert epidemiological models that forecast epidemic growth using baseline information about transmission (Chowell et al. 2016).

2. $n$ - the number of people physically present in the classroom, assumed to be constant across all classes for a given student or faculty member.

3. $i$ - the total number of class meetings in a term for the individual. For example, if a student has 12 class meetings per week for 12 weeks, $i=144$.

4. $\sigma$ - the probability of transmission to one or more persons in the classroom, given that an infected person is present. The probability of transmission in the classroom is considered to be different from the basic reproduction number that gives rise to the prevalence of infection in the community, $\lambda$, because the classroom is a special environment. For example, if classroom mitigation strategies are effective then probabilty of transmission in the classroom might be lower than in the general population.

Reducing the problem to these factors has the virtue of simplification (Kucharski 2020; Grawe 2020). Note that time is not included among these factors, because a decision must be made at the beginning of the term to teach in classrooms or online only. It is assumed it does not matter if a student is infected early in the term or late in the term; rather, the probability of interest is of infection resulting from a specific course of action (number of classes, number of class meetings, class size) given the prevalence of the disease in the community. Similarly, these factors do not account for the duration an individual is capable of transmitting, which for COVID-19 appears to be much longer than the time between class meetings, or the susceptibility (resilience) of individuals who encounter the disease in the classroom, although this term could be included.

The relationships among these factors can be considered in terms of the likelihood that an infected individual will be present in the classroom during the term and the likelihood that this individual will infect another person in the classroom. Scheduling in advance of the term requires that class size, $n$, and number of class meetings, $i$, be determined in advance. For a given prevalence of infection in the community, $\lambda$, we seek a probability of infection that approaches zero as $\lambda \rightarrow$ 0 or as the total number of classroom interactions approaches zero, $n i \rightarrow 0$. Additionally, as these values grow large, the probability of an infected individual in 
the classroom during the term should approach 1 asymptotically. An exponential relationship (equation 1 ),

$$
P=\sigma[1-\exp (-\lambda n i)]
$$

meets these boundary requirements to describe how dampening factiors $(\lambda, n, i)$ reduce the probability of infection $(P)$ from the probability of transmission $(\sigma)$.

The probability of transmission in the classroom, $\sigma$, is considered to be constant in the simplest model. It might also be considered a function of factors such as class size, i.e., $\sigma(n)$.

This model assumes that prevalence of COVID-19 in the community, $\lambda$, is constant. Expert epidemiological models are often concerned with forecasting $\lambda$ for a particular time period and a particular population, or with forecasting the rate of change in $\lambda$ with time. These expert models forecast future values of $\lambda$ using a combination of statistical models, analysis and data assimilation, where the data include past rates of infection - a strong argument for widespread testing. Prevalence in the community is also estimated based on the rate of hospitalization or death attributed to COVID-19. Some of these expert models attempt to incorporate other factors, such as social distancing measures and practice, and they have done an applaudable job of forecasting change in the prevalance of COVID19 in communities (e.g., cities, counties, nations) on timescales of weeks or months. ${ }^{1}$

While expert models are often concerned with forecasting the prevalence of new COVID-19 infections in a community, equation (1) uses these estimates to forecast the probability of an individual becoming infected as a result of classroom interaction, given this rate $(\lambda)$. For sure, the value of $\lambda$ will change with time, and epidemiologists will forecast these changes. From a quantitative literacy perspective, we should be prepared to use the information they provide to focus in and estimate the probability of students or faculty members becoming infected as a result of their interactions in university classrooms in the context of $\lambda$.

The probability of transmission, $\sigma$, is constant in equation (1), meaning that it does not depend on the number of students in the classroom or the number of interactions, or vary between classrooms. Of course, probability of transmission in a classroom environment really does vary depending on a host of factors, which include the size of the room, the airflow in the room, the length of class meeting

${ }^{1}$ Many models for forecasting rate of change in new COVID-19 cases in U.S. communities are currently summarized on Nate Silver's FiveThirtyEight website: "Where The Latest COVID-19 Models Think We're Headed - And Why They Disagree", by R. Best and J. Boice, URL: https://projects.fivethirtyeight.com/covid-forecasts/?cid=rrpromo (last accessed June 9, 2020). Specific models include the MIT COVID Analytics webpage: https://www.covidanalytics.io/, the Los Alamos model: https://covid-19.bsvgateway.org/, and Columbia University model: https://github.com/shaman-lab/COVID-19Projection, among others 
times, in addition to the number of students in class, to name a few factors. By holding $\sigma$ constant in this simple model, the variation in these factors is ignored, and it is acknowledged that a more nuanced view of probability of transmission is ultimately required. This simplification is useful, nevertheless, if one wants to calculate the change in probability, $P$, with change in the number of class meetings held during the term, assuming that conditions in the room during successive class meetings are similar enough to consider probability of transmission to be constant.

It is worth emphasizing that the probability of infection in the classroom, $P$, is directly proportional to probability of transmission, $\sigma$, and exponentially related to prevalence of the disease in the community and total number of classroom interactions, $\lambda, i$, and $n$. The reason for this mathematical relationship is that $\sigma$ itself is a conditional probability and so the unconditional probability, $P$, depends directly upon the value of $\sigma$. The other terms, $\lambda, i$, and $n$, are parameters in the exponential model used to calculate the probability that an infected person is present in class. In other words, $P$ is modeled as the product of two other probabilities. The latter probability is calculated using an exponential function because it must approach 1 asymptotically with increase in prevalence of infection in the community and total number of classroom interactions. Since prevalence of infection in the community, $\lambda$, is a rate (number of cases per number of persons in the entire community) and because class size, $n$, and number of class meetings, $i$, represent $i \times n$ independent trials, the probability model is discrete and exponential (also called a Poisson model, e.g., Larsen and Marks 1986).

These mathematical relationships have important implications for strategies to lower the probability of infection spread in classrooms among students. Specifically, for a given rate of infection among the classroom population, strategies to reduce class size $(n)$ or number of class meetings $(i)$ will not do a particularly effective job of reducing the probability of individuals becoming infected by classroom interactions. Instead, it is much more effective to reduce the probability of transmission in the classroom, because the probability is directly proportional to $\sigma$. Perhaps $\sigma$ might be reduced through measures such as improving airflow, social distancing (2-m offset), effective use of face masks and related proactive measures.

Consider a numerical example that illustrates these relationships:

1. $\lambda=0.0001$. The prevalence of infection in the university population is assumed to be 1 in 10,000. There is uncertainty in the actual value, of course. It varies among universities and will change with time, likely being updated with improved expert epidemiological models. The 1:10,000 value here is used only as an example. For comparison, the University of South Florida (USF) is located in a county of approximately 1.4 million people and has reported COVID-19 infections of about 2,100 people during March-May, 2020 , or an occurrence of between 1 in 1,000 and 1 in 2,000 with strong caveats due to insufficient testing. Using a value of 1 in 10,000 assumes a 
lower prevalence during the coming term among the USF population, which might be overly optimistic. Hence from a quantiative literacy perspective, it is important to understand this parameter and to re-calculate probability of infection in the classroom as new estimates emerge.

2. $n=0$ to 100 - the number of students physically present in a classroom. An online-only classroom is represented by $n=0$.

3. $i=144$ - the total number of class meetings in a term for the individual.

4. $\sigma_{1}=0.1$ - the probability of transmission in the classroom under normal circumstances without social distancing or related procedures.

$\sigma_{2}=0.05$ - the probability of transmission in an interior classroom with some social distancing rules or related procedures in place.

$\sigma_{3}=0.01$ - very effective social distancing in place, such as using outdoor classrooms, which reduces the probability of transmission in the classroom, given the presence of an infected individual, by one order of magnitude compared to transmission probability in a 'normal' classroom.

These numbers are for illustration only since the actual values are unknown or vary. The resulting probabilities of infection of one or more individuals in a classroom setting in Fall term are plotted in Figure 1.

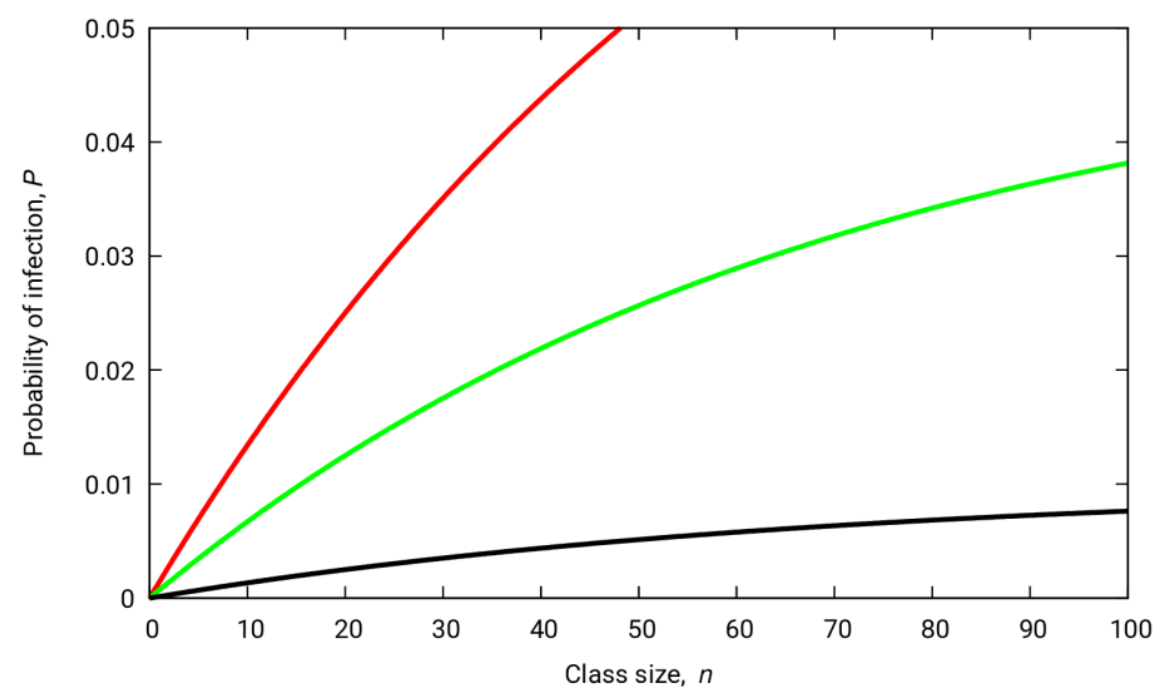

Figure 1. Probability of one or more students or faculty members being infected during Fall term as a function of class size, given constants described in text for three different transmission rates in the classroom. The three curves represent variation in probability estimates with varying transmissibility: red curve, $\sigma_{1}=0.1$; green curve, $\sigma_{2}=0.05$; black curve, $\sigma_{3}=0.01$. 
Next consider the same constants but vary the number of class meetings while holding class size constant (Figure 2). The two plots (Figures 1 and 2) are basically the same because the probability depends on $i \times n$, or total classroom interactions. Students, however, generally have a fixed number of classroom meetings per term and benefit from smaller class sizes. Faculty members are responsible for meeting student demand, so smaller class sizes mean more sections and the same number of total interactions. Duration of classroom meetings is not considered, but might also be factored into the estimate of $\sigma$.

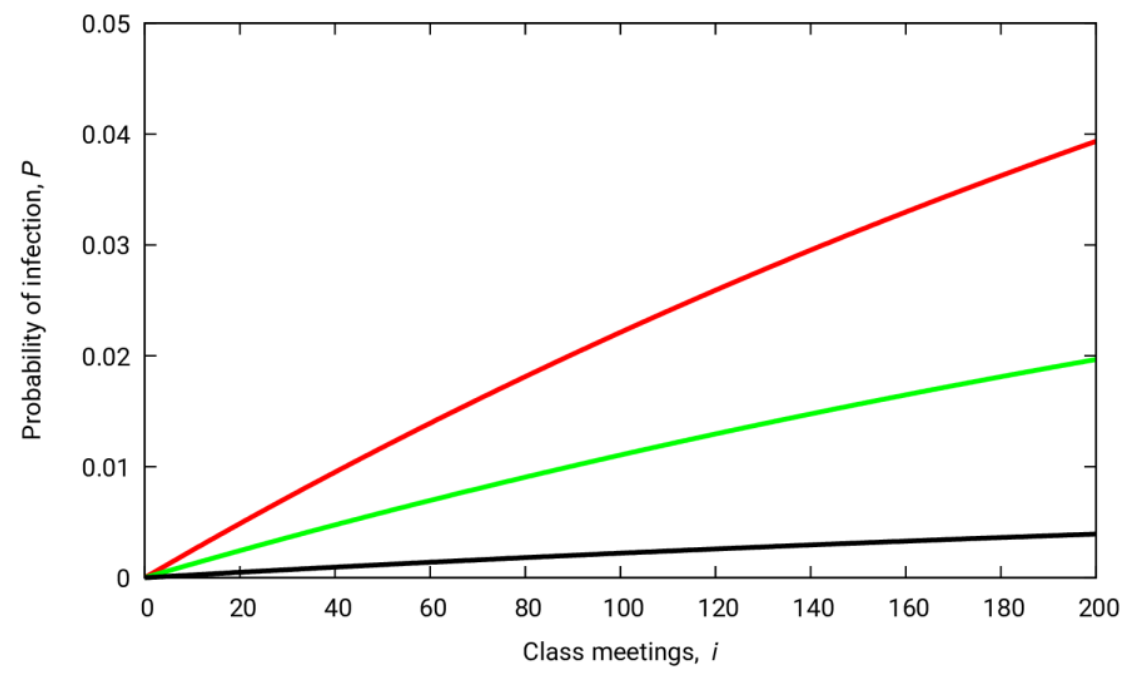

Figure 2: Probability of one or more students or faculty members being infected during Fall term as a function of total number of class meetings, given constants described in text for three different transmission rates in the classroom. In this example the class size is assumed to be $n=25$. The three curves represent variation in probability estimates with varying transmissibility: red curve, $\sigma_{1}=0.1$; green curve, $\sigma_{2}=0.05$; black curve, $\sigma_{3}=0.01$.

Regardless, the outcome is that it is quite difficult to substantially change the probability of an individual being infected through classroom interactions by changing the class size or number of class meetings. For example, changing the upper limit of class size from 100 to 50 has rather insubstantial impact. Only reducing class interactions dramatically - say limiting class size $<10$ or total class meetings during the term to say $<50$ (for maximum class size $<25$ ) - reduces the probability to $<0.01$ in this example for relatively high values of $\sigma$, the probability of transmission in the classroom. This places a strain on hybrid models because, for a given transmission rate, these approaches are effective only if class meetings are quite small and quite infrequent. Rather, it is critical to reduce the probability of transmission in the classroom. 


\section{A More Complex Model}

A big assumption in the simple model is that the probability of transmission given an infected individual is present in the classroom, $\sigma$, is a constant. Of course, it is likely that $\sigma$ also depends on class size assuming that social distancing and related guidelines are easier to follow in a less crowded classroom.

Consider a relationship between the effectiveness of transmission and class size:

$$
\sigma(n)=\sigma_{1}+\left(\sigma_{2}-\sigma_{1}\right)\left[1-\exp \left(-\frac{n-1}{v}\right)\right]
$$

where $\sigma(n)$ is the probability of transmission in the classroom, which now depends on the number of individuals in the class $(n) ; \sigma_{1}$ is the ideal (low) transmissibility in the classroom with few individuals and $\sigma_{2}$ is the upper limit of transmissibility, which might be the value if no social distancing is effectively occurring due to large class size.

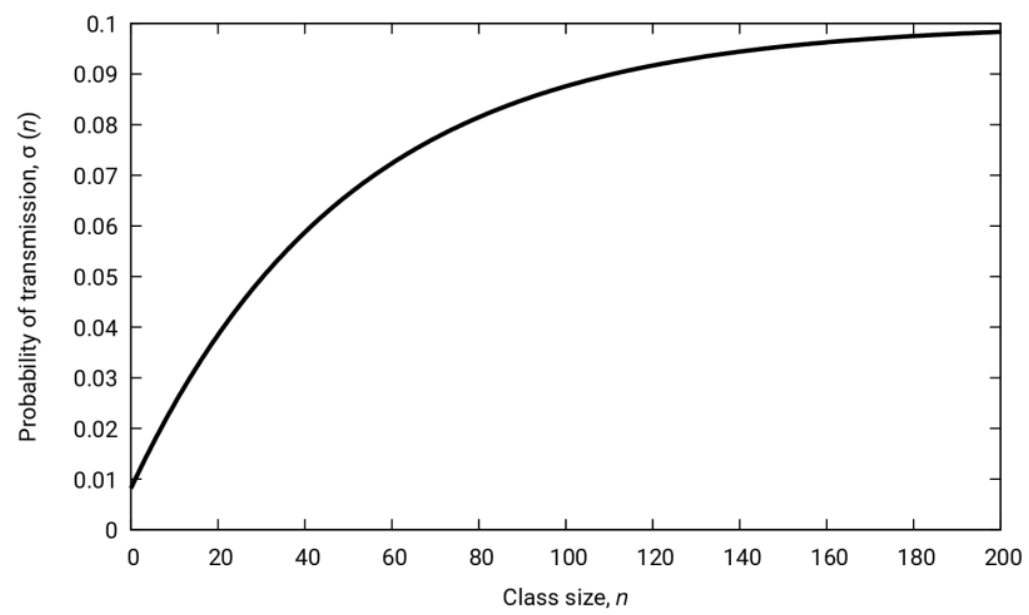

Figure 3. A relationship between probability of transmission, $\sigma(n)$, and class size, $n$, assuming a rate constant $v=50$ persons, low transmission probability of $\sigma_{1}=0.01$ and high transmission probability of $\sigma_{1}=0.1$.

The constant $v$ in the denominator is a capacity factor, in units of persons (students and faculty members), that scales change of $\sigma(n)$ with change in class size. It is not a room capacity, at least in the normal sense, because it depends on more than the number of seats in the room or the number of exits. This capacity factor, $v$, depends on the distance between seats, the way in which the room is maintained, the nature of the air-handling system and airflow, the behavior of classroom participants with regard to social distancing, and all other factors that affect the probability of transmission during a class meeting, bounded by limits $\sigma_{1}$ and $\sigma_{2}$. 
The term $n-1$ in the numerator reflects the number of people present in the classroom to whom the virus can be transmitted, not including the individual who is carrying the infection. For example, suppose a lecture hall has a maximum room capacity of 200 students. If $v=50$ persons and $n=200$ students, $\sigma(n)$ is about equal to $\sigma_{2}$, and there is effectively no social distancing in the lecture hall. Reduce class size to $n=50$ or $25 \%$ of the normal room capacity, then $\sigma(n) \approx 0.07$, a substantial improvement in probability of transmission but not as good as could be achieved by further reduction in class size (Fig. 3). The parameter $v$ in equation (2) plays the same role as $\lambda$ in equation (1), but in this case is shown as a reciprocal rate so that the units of $v$ are persons (students and faculty members).

Given this functional relationship between transmissibility $(\sigma)$ and class size $(n)$, the probability of a student being infected during the Fall term becomes:

$$
P=\sigma(n)[1-\exp (-\lambda n i)]
$$

As before, we can explore the variation in probability of a individual student or faculty member being infected during Fall term as a function of class size.

1. $\lambda=0.0001$. The rate of infection in the university population is assumed to be 1 in 10,000, as before.

2. $n=0$ to 100 - the number of students in a classroom, as before.

3. $i=144$ - the total number of class meetings in a term for the individual, as before.

4. $\sigma_{1}=0.01$ - the probability of transmission in the classroom with few students; $\sigma_{2}=0.1$ - the probability of transmission in a classroom with many students, given that an infected student is present in the classroom.

5. $v_{1}=10, v_{2}=50, v_{3}=100$ to explore the effect on $P$ of changing transmissibility as a function of class size.

One can see from a plot of these relationships (Fig. 4) that probability of an individual becoming infected during the Fall term is strongly dependent on class size if transmissibility is a function of class size (e.g., larger classes are less likely to achieve social distancing and related measures to limit transmission). Note that introduction of a probability of transmission that depends on class size scaled by capacity factor makes the relationship between class size and probability of infection more nearly linear, with the change in probability as a function of class size depending on the assumed value of the capacity factor, $v$. 


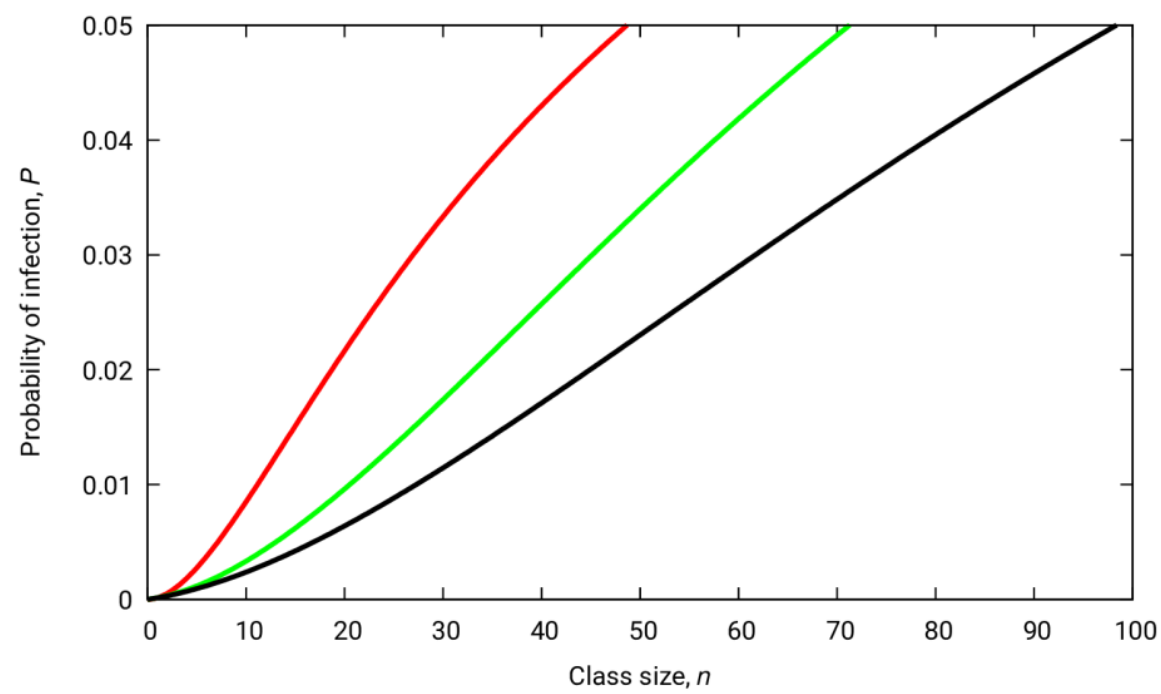

Figure 4. Probability of one or more students or faculty members being infected during Fall term as a function class size, if transmissibility, $\sigma(n)$, is a function of class size. Red curve, $v_{1}=10$; green curve, $v_{2}=50$; black curve, $v_{3}=100$. In this example, low transmission probability is $\sigma_{1}=0.01$, and high transmission probability is $\sigma_{2}=0.1$.

\section{Limitations and Implications}

These models do not capture the intricacies of disease spread in the community, which is the purview of expert models. Specifically, the infection rate in the university community is assumed to be random throughout the term. Clearly, disease clusters might develop, possibly driven by 'super spreaders' (Ndairou et al. 2020) and alter the probability of infection for individuals in particular areas. The two models presented here are better treated as the probability of first infection in the classroom, with the hope that some response would change the probability thereafter.

Testing students and faculty may result in much better understanding of the prevalence of infection within the university community, $\lambda$, or hopefully eliminate some infected individuals from the classroom while they are contagious. Even the average rate of infection will likely not be constant throughout a term. Some U.S. universities, for example, are anticipating an increase in prevalence of infection in the community, $\lambda$, associated with Fall Thanksgiving holiday and may ban classroom activities after that holiday. If one anticipates that $\lambda$ will decrease as the term progresses, block scheduling might also be an effective strategy for limiting risk. In block scheduling, different classes are taken at an accelerated rate for shorter periods of time. One strategy is to front-load the term with courses that are reasonably taught online, saving lab courses for later in the term. As these examples 
are meant to illustrate, as we are updated by experts with new estimates of the prevalence of infection in the community we can use that new information (equations 1-3) to estimate affects on the classroom and take action.

It is assumed in these models that class size and class meetings are independent variables but some students likely attend more than one class together. In fact, the probability of infection in a classroom would be reduced if the same cohort of students attended all classes together, because in that case these variables would have joint variation. Cohorts might be practical for some students, especially upperclassman taking courses within their majors. For example, there might be a cohort of geology or environmental biology students taking their courses as a group with the goal of decreasing the number of independent classroom interactions. This strategy would not appear to work for students taking a large number of service courses.

The change in probability of transmission as a function of class size is not well known and may even be better represented as steps or thresholds. On the other hand, these simple exponential models do indicate that the change in transmission with class size is an important consideration. As social distancing and all it entails will likely be part of the strategy of most universities, one might quantify the relative effectiveness of specific measures by considering the effect of these measures on the capacity factor, $v$.

Some students may have immunity or reduced susceptibility if they have already been exposed to the virus. The number of students with immunity is unknown but, if estimated, might be accommodated by reducing the class size variable, $n$, to an effective class size reflecting the number of persons present in class who are susceptible. Alternatively, an additional susceptibility factor might be introduced. As with class size and number of class meetings, presence of individuals with low or no susceptibility has very little impact on the overall probability until the number of immune individuals approaches the total number in class.

To apply a probability model to make a decision, one requires a threshold. Is $P=0.01$ for the probability of an individual being infected by classroom interaction during the term sufficiently low to allow classroom activities on campus? Or should the threshold for classroom activities be $P=0.001$ ? As epidemiological models improve their resolution, how is information about the prevalence of COVID-19 in a given university community best used to make decisions about classroom meetings? The appropriate probability thresholds likely depend on university size. Larger universities with many classes likely want to choose a lower threshold probability since the total number of classroom interactions is high. Faculty and students might choose their threshold number of classroom interactions depending on their personal situations. An advantage of application of probabilistic models is that they elevate discussion about thresholds among the community, so that the consequences of decisions are potentially more 
widely understood by participants. These discussions need to include uncertainty, which for some model parameters is high (e.g., $\sigma(n)$ and $v$ in these models). Here uncertainty has been partially explored using alternative parameter values.

\section{Conclusions}

The example probabilities calculated here are meant only for purposes of comparison, since so many of the rates and model parameters are unknown. The comparison suggests:

1. Hybrid (online with limited class meetings) will not be particularly effective for reducing the probability of an individual being infected during the term unless class meetings are highly limited (e.g., <10 per term) and limited to few participants. In other words, the disease might be sufficiently prevalent in the community that few interactions are needed because the total number of student and faculty interactions in a university community is so large. This implication, in turn, suggests a strategy of online teaching exclusively is best, or a strategy must be developed and adhered to in which transmission can be minimized given the presence of an infected person in the classroom.

2. Given any form of classroom teaching (normal teaching schedules or hybrid models), the probability of infections is strongly dependent on the effectiveness of social distancing and related strategies for reducing transmission in the classroom (e.g., optimizing airflow, plexiglass shields, cleaning between classes). This implication arises because the probability is directly proportional to the probability of transmission, $\sigma$ or $\sigma(n)$ in the presented models.

3. Effectiveness of social distancing and related measures to reduce transmission in the classroom probably depend on class size. This rate dependence is unknown but effectively limits class size to situations in which transmission can be maintained at low and reasonably constant levels.

Overall, these results seem to indicate that the most effective strategy for reducing transmission in the classroom during the pandemic will be to reduce the number of students in classrooms to well below the room capacity, maintain clean facilities, use facilities that limit transmission (e.g., consider outdoor seating where practicable, avoid windowless lecture halls with poor airflow) and strongly encourage individuals to adhere to guidelines to reduce classroom transmission. That places demands on university infrastructure and on the university community. Whether classroom teaching is feasible in the coming term depends on whether these demands can be met. 
As a teacher, I present my students with quantitative models and encourage them to achieve model literacy (Courtland et al. 2012). Regardless of the teaching format I adopt this coming term, my students and I will likely begin by evaluating probabilistic hazard models that have lately been useful in forming our decisions. A core message of quantitative literacy is that we all must develop these skills to handle the critical challenges in our lives.

\section{Acknowledgments}

I thank Len Vacher, not only for his encouragement with this manuscript but for the thoughtful perspective he has brought to our discussions on numeracy for many years. Timely comments by four anonymous revewers improved this manuscipt. Thank you! Additional comments on the text by Illiana Braums, Joni Downs, Steven Reader, Pritish Mukherjee, Nathan Grawe and Laura Connor were very helpful.

\section{References}

Chowell, G., L. Sattenspiel, S. Bansal, C. Viboud. 2016. "Mathematical Models to Characterize Early Epidemic Growth: A Review." Physics of Life Reviews 18: 66-97. https://doi.org/10.1016/j.plrev.2016.07.005

Connor, C.B. 2011. "A Quantitative Literacy View of Natural Disasters and Nuclear Facilities.” Numeracy 4(2): Aticle 2. http://doi.org/10.5038/19364660.4.2.2

Courtland, L., C.B. Connor, L.J. Connor, C. Bonadonna. 2012. "Introducing Geoscience Students to Numerical Modeling of Volcanic Hazards: The Example of Tephra2 on VHub. org." Numeracy 5(2): Article 6. http://doi.org/10.5038/1936-4660.5.2.6

Grawe, Nathan D. 2020. "From $R_{0}$ to the Herd: A Review of The Rules of Contagion, by Adam Kucharski." Numeracy 13(2): Article 10. https://doi.org/10.5038/1936-4660.13.2.1363

Kafka, A.C. 2020. "Why the Fall Will Be a Liability Minefield." Chronicle of Higher Education, May 29.

Kucharski, Adam. 2020. The Rules of Contagion: Why Things Spread-and Why They Stop. London: Profile Books, Ltd.

Larson, R.J., and M.L. Marx. 1986. An Introduction to Mathematical Statistics and Its Applications. Englewood Cliffs: Prentice-Hall.

Levantovskaya, M. 2020. "Faculty are Not Cannon Fodder." Chronicle of Higher Education, May 28.

Manski, C.F., F. Molinari. 2020. "Estimating the COVID-19 Infection Rate: Anatomy of an Inference Problem." Journal of Econometrics 4(41). May 6. https://doi.org/10.1016/j.jeconom.2020.04.041. 
Ndairou, F., I. Area, J.J. Nieto, D.F.M. Torres. 2020. "Mathematical modeling of covid-19 transmission dynamics with a case study of Wuhan." Chaos, Solitons \& Fractals. 35:109846. https://doi.org/10.1016/j.chaos.2020.109846.

Vacher, H.L., D. Wallace. 2008. “The Scope of Numeracy.” Numeracy 1(1): Article 1. http://doi.org/10.5038/1936-4660.1.1.1

Vacher, H.L. and N.D. Grawe. 2019. "Roots and Seeds: Finding Our Place in the Social Practice Nexus That Is Quantitative Literacy." Numeracy 12(2):

Article 1. https://doi.org/10.5038/1936-4660.12.2.1

Wallace, D. 2020. "Three Formative Questions in the Quantitative Literacy Movement." Numeracy 12(1): Article 13. https://doi.org/10.5038/1936$\underline{4660.12 .1 .13}$ 